\title{
Conformal invariance and its breaking in a stochastic model of a fluctuating interface
}

\author{
Francisco C. Alcaraz ${ }^{1}$, Erel Levine ${ }^{2}$ and Vladimir \\ Rittenberg ${ }^{3,4}$ \\ ${ }^{1}$ Instituto de Física de São Carlos, Universidade de São Paulo,Caixa Postal 369, \\ 13560-590 São Carlos, SP, Brazil \\ ${ }^{2}$ Center for Theoretical Biological Physics, University of California at San \\ Diego, La Jolla, CA 92093 \\ ${ }^{3}$ Department of Mathematics and Statistics, University of Melbourne, Parkville, \\ Victoria 3010, Australia \\ ${ }^{4}$ Physikalisches Institut, Bonn University, 53115 Bonn, Germany.
}

\begin{abstract}
Using Monte-Carlo simulations on large lattices, we study the effects of changing the parameter $u$ (the ratio of the adsorption and desorption rates) of the raise and peel model. This is a nonlocal stochastic model of a fluctuating interface. We show that for $0<u<1$ the system is massive, for $u=1$ it is massless and conformal invariant. For $u>1$ the conformal invariance is broken. The system is in a scale invariant phase with the dynamical critical exponent $z$ and other critical exponents varying continuously with $u$. As far as we know it is the first example of a system which shows such a behavior. Moreover in the broken phase, the critical exponents vary continuously with the parameter $u$. This stays true also for the critical exponent $\tau$ which characterizes the probability distribution function of avalanches (the critical exponent $D$ staying unchanged).
\end{abstract}

\section{Introduction}

The raise and peel model (RPM) of a fluctuating one-dimensional interface was introduced in Ref. [1]. In this model, the evolution of the interface (described in terms of RSOS paths), in the presence of a rarefied gas of tiles (tilted squares or particles), follows Markovian dynamics. When a tile hits a site of the interface it can be reflected, adsorbed with a probability $u_{a}$ ("raising" the interface) or trigger a desorption process with a probability $u_{d}$, depending on the heights of the two neighbouring sites. In the desorption process part of the top layer of the interface evaporates (the interface is "peeled"). The number of tiles which leave the interface in one desorption process, which may be macroscopic, define the size of an avalanche.

The model depends on a single parameter $u=u_{a} / u_{d}$ or alternatively $w=1 / u$. The case $u=1$ was discussed in detail in [1] in the continuum time limit. There are two reasons why this case has received so much attention:

a) The time evolution of the system is given by a Hamiltonian $H$ which, in the finite-size scaling limit, has a known spectrum. The reason is that in this limit $H$ describes a $c=0$ conformal field theory (CFT) ( $c$ is the central charge of the Virasoro algebra) and the spectrum is given by characters of this algebra [2]. Moreover this observation implies that the dynamical critical exponent is $z=1$. 
b) The weights of the various RSOS paths in the probability distribution function (PDF) of the stationary state for a finite system are related to a counting problem of alternating sign matrices with different topologies (see [3] and references therein). Alternating sign matrices have miraculous combinatorial properties [4] and this has allowed to guess (conjecture) the expressions of several physical quantities in the stationary state for any number of lattice sites based on exact calculations for small lattices.

In [1] very little work was done for $u$ different of one except for a crude estimate of the phase diagram. The reason is simple: for $u=1$ one has used the exact knowledge of the stationary state for lattice sizes up to 18 sites (it is hard, although possible, to go beyond this number) and made (unproven) conjectures for any lattice sizes. For $u \neq 1$, one has only diagonalized numerically (up to 14 sites) the Hamiltonian, that gives the time evolution of the system, in order to estimate in which domain of $u$ the system is massless (scale invariant) or massive (finite correlation lenghts). The results were inconclusive.

In this paper we present an extensive study of the model using Monte Carlo simulations. What was our motivation?

a) For $u=w=1$, the RPM is, to our knowledge, the first example of a stochastic process in which conformal invariance, a time-space symmetry, plays a role and one is interested to see some consequences. Let us stress that this is different of the one timetwo space abelian sand-pile model where conformal invariance is seen in the stationary state [5] We also wanted to check the conjectures made in [1] for large lattice sizes and to study other properties of the model.

b) When $u=1 / w \neq 1$ several scenarios are possible. The system may stay conformal invariant, may get massive or stay scale invariant without being conformal invariant. According to the standard lore (see Refs. [6] and [7] for a more precise definition of the conditions) provided that the interactions are local, scale invariance implies conformal invariance. Although for $u=w=1$, the RPM can be mapped onto the spin zero sector of an $U_{q}(s l(2))$ invariant XXZ quantum chain [1] in which the interaction is local, as far as we can see, for $u \neq 1$ a mapping onto a system with local interactions is not possible. Therefore, keeping in mind that the desorption process is not local, for $u \neq 1$ we still have the interesting possibility that the system can be scale invariant without being conformal invariant.

We will show that for $u<1$ the system is massive while for $u>1(w<1)$ the system is scale invariant but not conformal invariant, with the critical exponent $z$ [8] and other critical exponents varying continuously with $w$. At $w=0$ the system becomes massive again. We think that it is for the first time, that such a phenomenon is seen.

c) As noticed already in Ref. [1] and confirmed in the present paper, for $u=1$ the probability distribution function (PDF) of avalanches triggered by tiles hitting the interface in the stationary state, has a long tail, the critical exponents being $D=1$ and $\tau=3$ (see Section 5 and Ref. [11] for definition of the exponents), which means that the model exhibits self-organized criticality (SOC). We will show how the PDF of avalanches behave if one varies $w$.

The paper is organized as follows. In Section 2, following [1] we present the model and define the quantities which we found useful to characterize the interface. Those are contact points, clusters, heights and the fraction of sites of the interface where desorption does not take place (FND), i.e. the density of local maxima or minima.

In Section 3 we describe the properties of the stationary states for various values 


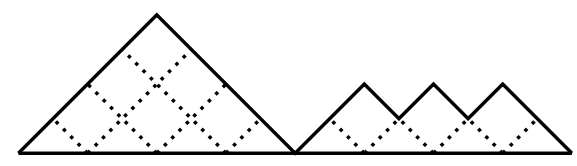

Figure 1. A configuration of the interface with three contact points and two clusters for the lattice size $L=16$.

of $u$, and derive the phase diagram of the model (Fig. 4). We give the variation of the number of clusters with the size of the system $L$. We show that for $u<1$ the density of clusters is finite for large values of $L$. For $u=1$ this density vanishes like $L^{-\frac{1}{3}}$. For $w<1$ the density of clusters also vanishes as a power law with an exponent which varies with $w$. We also give the local density of contact points as a function of the size of the system and its finite-size scaling behavior. We discuss the size dependence of the average height. The variation of the FND with $w$ and the system sizes is given in Section 5.

In Section 4 we give the values of the dynamical critical exponent $z$ for various values of $w$. We estimate $z$ using the Family-Vicsek scaling for various observables. We show that $z$ decreases from the value 1 for $w=1$ towards 0 at small values of $w$. For $w=1$ we show how the knowledge of the finite-size spectrum (explained in appendix A) gives information on the Family-Vicsek scaling functions. We also give the two-contact points correlation function obtained using Monte-Carlo simulations and illustrate the predictions of conformal invariance.

In Section 5 we study the PDF of avalanches for different values of $w$. The average size of the avalanches stays finite in the whole domain $1>w>0$ and its value is given remarkably well by mean field. We show that the exponent $D$ is equal to 1 for all values of $w$ but that the exponent $\tau$ [11] changes from the value 3 at $w=1$ to a value close to 2 for smaller values of $w$. Our conclusions are presented in Section 6 .

\section{The raise and peel model}

We consider a one-dimensional lattice with $L+1(L=2 n)$ sites. An interface is formed by attaching at each site non-negative integer heights $h_{i}(i=0,1, \ldots, L)$ which obey the restricted solid-on-solid (RSOS) rules:

$$
h_{i+1}-h_{i}= \pm 1, \quad h_{0}=h_{L}=0, \quad h_{i} \geq 0 .
$$

There are $C_{n}=(2 n) ! /\left((n+1)(n !)^{2}\right)$ possible configurations of the interface. In Fig. 1] we show a configuration for $n=8(L=16)$. Alternatively, one can describe the interface using slope variables $s_{i}=\left(h_{i+1}-h_{i-1}\right) / 2,(i=1, \ldots, L-1)$.

The dynamics of the interface is described in a transparent way in the language of tiles (tilted squares) which cover the area between the interface and the substrate $\left(h_{2 i}=0, h_{2 i+1}=1,(i=0, \ldots, n)\right)$ (see Fig. (1).

We consider the interface separating a film of tiles deposited on the substrate from a rarefied gas of tiles. We are interested to find the evolution of the interface towards the stationary state and to study the properties of the interface in this state.

The evolution of the system in discrete time (Monte-Carlo steps) is given by the following rules. With a probability $P_{i}=1 /(L-1)$ a tile from the gas hits site $i,(i=1, \ldots L-1)$. Depending on the value of the slope $s_{i}$ at the site $i$, the following processes can occur: 


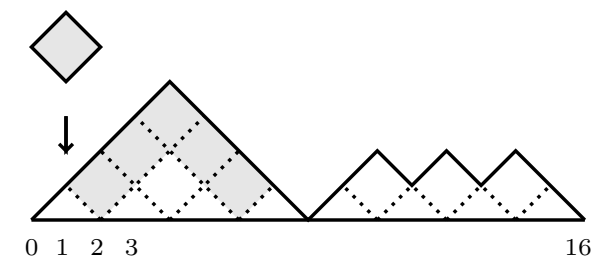

Figure 2. A desorption event. The incoming tile at site 1 triggers, with probability $u_{d}$, an avalanche of 5 tiles, which are shaded. All of the shaded tiles are removed in the desorption event.

i) $s_{i}=0$ and $h_{i}>h_{i-1}$.

The tile hits a local peak and is reflected.

ii) $s_{i}=0$ and $h_{i}<h_{i-1}$.

The tile hits a local minimum. With a probability $u_{\text {a }}$ the tile is adsorbed $\left(h_{i} \mapsto h_{i}+2\right)$ and with a probability $1-u_{\mathrm{a}}$ the tile is reflected.

iii) $s_{i}=1$.

With probability $u_{\mathrm{d}}$ the tile is reflected after triggering the desorption of a layer of tiles from the segment $\left(h_{j}>h_{i}=h_{i+b}, j=i+1, \ldots, i+b-1\right)$, i.e. $h_{j} \mapsto h_{j}-2$ for $j=i+1, \ldots, i+b-1$. This layer contains $b-1$ tiles (this is always an odd number). With a probability $1-u_{\mathrm{d}}$, the tile is reflected and no desorption takes place. For an example see Fig. 2 .

iv) $s_{i}=-1$.

With probability $u_{\mathrm{d}}$ the tile is reflected after triggering the desorption of a layer of tiles belonging to the segment $\left(h_{j}>h_{i}=h_{i-b}, j=i-b+1, \ldots, i-1\right)$, i.e. $h_{j} \mapsto h_{j}-2$ for $j=i-b+1, \ldots, i-1$. With a probability $1-u_{\mathrm{d}}$ the tile is reflected and no desorption takes place.

In the Monte Carlo simulations we choose $u_{d}=1$ and $u_{a}=u$ for $u=u_{a} / u_{d} \leq 1$, and $u_{d}=w, u_{a}=1$ for $u>1$. For convenience we will use either $u$ or $w$ as the free parameter of the problem. We use these notations since we consider below either very small values of $w$ (or $u$ ) and our intuition can be helped by the fact that for $u=0$ or $w=0$ the properties of the system are known. Namely, for both $u=0$ and $w=0$ the spectrum is massive [1], the stationary states being the substrate $(u=0)$ and a full triangle $(w=0)$.

It is useful to define some quantities which characterize the surface. An obvious geometric observable is the set of sites $j$ ( $j$ even) for which $h_{j}=0$ (the sites 0 and $L$ always belong to this set). These sites are also called contact points. This set is important, for example, to study desorption. Desorption events are limited to the area

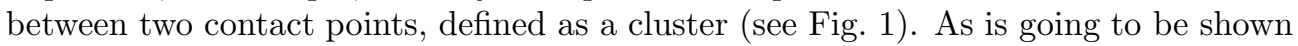
in Section 3, the density of contact points gives a local observable for which one can define various correlation functions (see also Section 4).

We first define quantities in the stationary state. The average number of clusters $k(L)$ and the density of clusters $\rho(L)$ are defined by:

$$
\begin{aligned}
& k(L)=\left\langle\sum_{j=1}^{L} \delta_{h_{j}, 0}\right\rangle, \\
& \rho(L)=k(L) / L .
\end{aligned}
$$



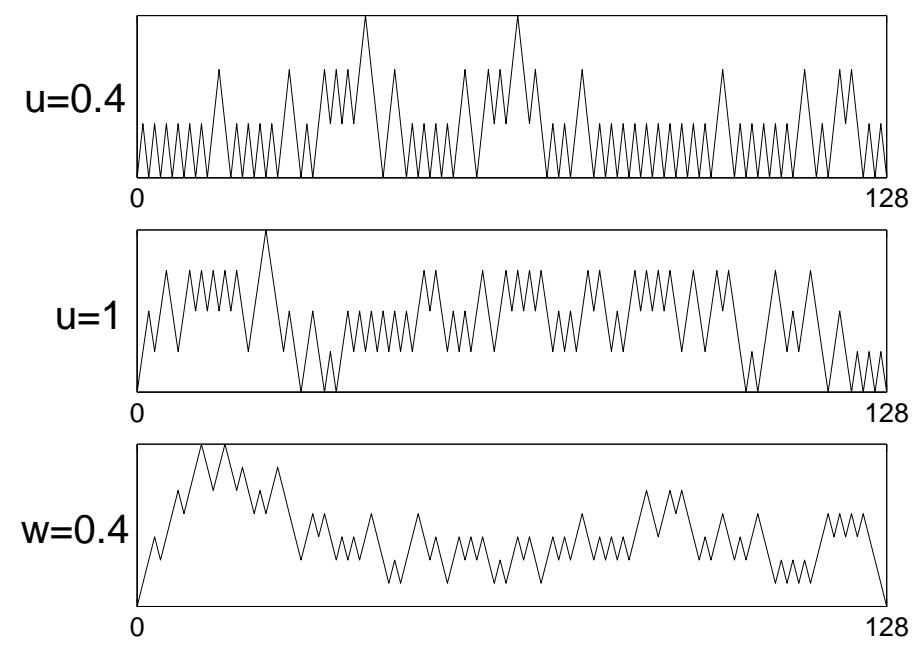

Figure 3. Typical configurations in the stationary states for $L=128$ sites and three values of $u$.

Notice that the maximum value of $\rho(L)$ is $\frac{1}{2}$ which is obtained when $u=0$ and one has only one configuration in the system - the substrate. The average height is defined as:

$$
h(L)=\frac{\left\langle\sum_{j=1}^{L} h_{j}\right\rangle}{L} .
$$

The lowest value of $h(L)$, corresponding to the substrate, is $\frac{1}{2}$. It is useful to consider also the average height in the middle of the system:

$$
h_{\frac{1}{2}}(L)=\left\langle h_{\frac{L}{2}}\right\rangle .
$$

A relevant quantity is the average of the fraction of the interface where desorption does not take place (FND)

$$
n(L)=\frac{1}{L-1}\left\langle\sum_{j=1}^{L-1}\left(1-\left|s_{j}\right|\right)\right\rangle .
$$

This quantity will be used in Section 5 since it allows to estimate the average number of tiles desorbed in avalanches. The large $L$ behavior of any of these quantities, say $h(L)$, will be denoted $h_{\infty}$ :

$$
h_{\infty} \equiv \lim _{L \rightarrow \infty} h(L) .
$$

In Section 4 we are going discuss average quantities which are not only $L$ dependent but also time dependent. For these quantities we use, for example, the notation $k(t, L)$ to denote the number of clusters.

\section{The stationary states of the RPM}

In order to get an intuition on the behavior of the model when $u$ changes, in Fig. 3 we show for $L=128$ typical configurations in the stationary states for three values of the parameter $u$. One notices that for $u=0.4$ there are many clusters, fewer for $u=1$ 


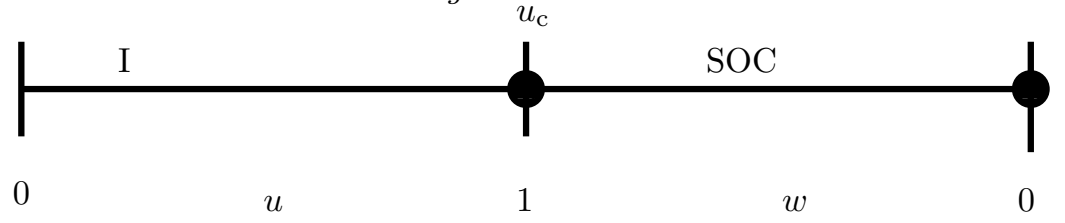

Figure 4. Phase diagram of the raise and peel model. For $0 \leq u<1$ (phase I), the model is massive. At $u=u_{c}=1$ it is massless and conformal invariant. For $1>w>0$ it is scale invariant with varying critical exponents and exhibits SOC. For $w=0$ the system is massive.

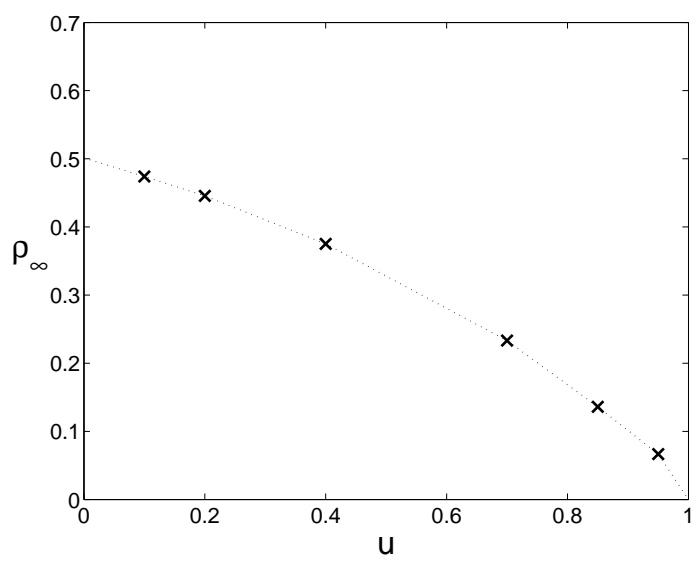

Figure 5. The density of clusters $\rho_{\infty}$ for various values of $u<1$.

and a single one for $w=0.4$ (other, less probable, configurations do exhibit several clusters). We also notice that the average heights are quite small. The Monte Carlo simulations were done for various lattice sizes up to $L=65536$ sites.

Before presenting our results, in Fig. 4 we show what be believe is the phase diagram of the model. In the domain $0<u<1$ the system is massive undergoing a second order phase transition at $u=u_{c}=1$. For $1>w>0$ the conformal symmetry seen at $w=1$ is broken but the system stays scale invariant. Since in this domain one has avalanches we call this phase a SOC phase. Another phase transition occurs at $w=0$ where the system becomes massive again. In the present section and in the next two ones we will give arguments which favor our conclusion.

We first consider the density of clusters $\rho(L)$ in the domain $0 \leq u<1$. Taking large values of $L$, one obtains the $u$ dependence of $\rho_{\infty}$ shown in Fig. 5 In all this domain the density of clusters stays finite. The density of clusters decreases from its maximum possible value $1 / 2$ which corresponds to the substrate at $u=0$ to the value zero for $u=1$. This indicates that there is a phase transition at $u=1$. From our Monte Carlo simulations it was hard to obtain the critical exponent which gives $\rho(u)$ when $u$ approaches the value one.

The local density of contact points $g(x, L)$ for $u=0.4$ and different lattice sizes is shown in Fig. 6(a). One observes that for small values of $x$, and large values of $L$, $g(x, L)$ decreases exponentially from the value 1 at $x=0$ (not shown on Fig. [6(a)) to a constant value in the bulk $\ddagger$.

$\ddagger$ This value is twice that seen in Fig. $[5$ since for even $x$ one always have $g(x)=0$. 

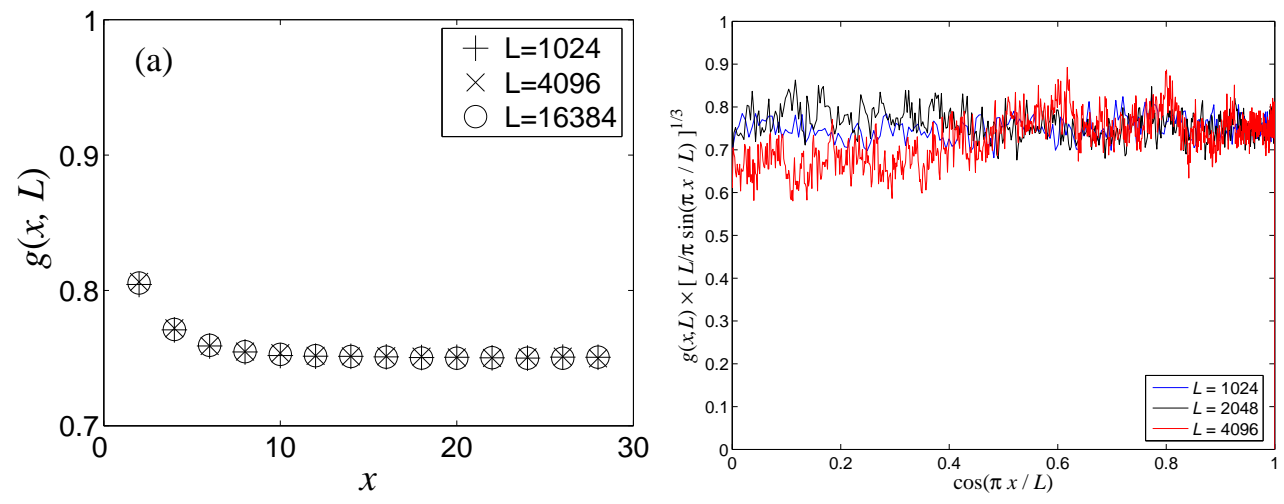

Figure 6. (a) The local density of contact points $g(x, L)$ at $u=0.4$ for different lattice sizes $L$. (b) The scaling function $C(\cos (\pi x / L))$ defined in 10 for $u=1$.

If we keep in mind that for $u=0$ one can diagonalize the related Hamiltonian of the stochastic process and show rigorously [1] that the system is massive, we can expect that the system is massive in the whole domain $0 \leq u<1$.

We turn now to the interesting case $u=1$. In Ref. [14] a conjecture was made which gives the probability to have $k$ clusters in a system of size $L$. For our purpose, we need only the behavior of the average number of clusters (see [1] Eq.(47)) for large system sizes, given by

$$
k(L) \simeq \frac{\Gamma(1 / 3) \sqrt{3}}{2 \pi} L^{\frac{2}{3}},
$$

which shows that the density of clusters vanishes algebraically for $u=1$. We have checked this conjecture using Monte Carlo simulations up to a system of size $L=512$. Since, as we show below, the density of clusters also vanishes algebraically in the entire domain $w<1$, it is convenient to define a critical exponent $\alpha<1$ which gives the power law increase of the number of clusters with the system size, $k(L) \sim L^{\alpha}$. For $u=1$ we have obviously $\alpha=2 / 3$.

We have considered, again for $u=1$, the density of contact points $g(x, L)$ for several lattice sizes and noticed that for the function

$$
G(x, L)=L^{\frac{1}{3}} g(x, L)=G(y),
$$

where $y=x / L$ one has a data collapse. We make an ansatz about the functional behaviour of $G(y)$ taking:

$$
g(x, L)=\left[\frac{L}{\pi} \sin (\pi x / L)\right]^{-1 / 3} C(\cos (\pi x / L)) .
$$

The motivation of this ansatz will become clear in the next lines. In Fig. 6 (b) one shows the function $C(\cos (\pi x / L)$. It is practically a constant. If one assume that $C$ is independent of $\pi x / L$, one can get its value by integrating $g(x, L)$ (Eq. (10) ) and compare the result with the average number of clusters given by (8). One obtains:

$$
C=-\frac{\sqrt{3}}{6 \pi^{\frac{5}{6}}} \Gamma\left(-\frac{1}{6}\right)=0.753149 \ldots,
$$

in very good agreement with the data (see Fig. 6(b)). 

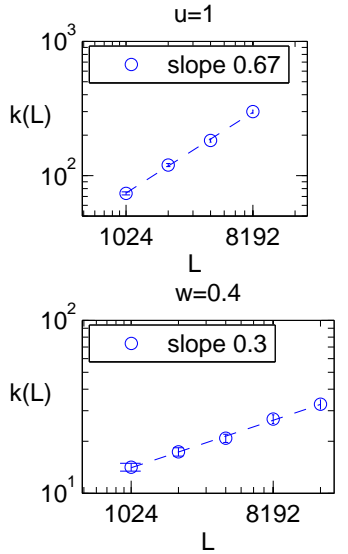

$w=0.85$

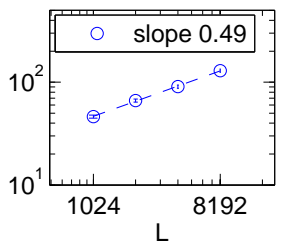

$w=0.1$

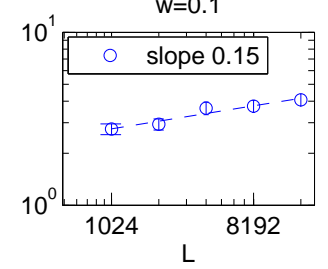

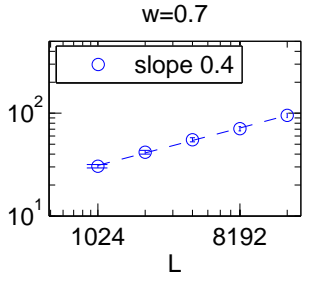

Figure 7. Average number of clusters $k(L)$ as a function $L$ for various values of $w$. The values of the exponent $\alpha$ (slope) is also shown.

If the density of contact points corresponds to a local operator in conformal field theory, one expects [15],

$$
g(x, L)=C\left[\frac{L}{\pi} \sin (\pi x / L)\right]^{X},
$$

where $X=\Delta+\bar{\Delta}$ is the scaling dimension of the local operator $(\Delta-\bar{\Delta}$ is the conformal spin). Comparing Eqs. (10)-(12) one concludes that the density of contact points corresponds to a local operator with scaling dimensions $X=1 / 3$. This result is surprising. One can show that the profile of an operator with spin vanishes [15]]. This implies $\Delta=\bar{\Delta}=1 / 6$. This value can't be obtained from the possible values of $\Delta$ (see (A.8) of minimal models. It might be obtained from $W\left(A_{n}\right)$ algebras $[16,17]$ but it is not clear why W-symmetry should play any role in our problem. Another possible explanation is that for a conformal theory with $c=0$ (this is also the case of percolation), under certain circunstances, the scaling dimension can be an arbitrary number $[18,19]$. This possibility is a bit strange since the number one has to obtain is a neat $1 / 6$. Finally, it is possible, that the proof that operators with spin have no profile functions does not apply to our problem. If this is the case, one can choose $\bar{\Delta}=0$ and $\Delta=1 / 3 \S$. The value $1 / 3$ is a perfectly acceptable one (see Appendix A). To conclude, we have not yet an explanation for the value $X=1 / 3$ in Eq. (12).

We move away from $u=1$ and consider the domain $1>w$. We observe that in Fig. $[7$ the number of clusters $k(L)$ keep having an algebraic increase with an exponent $\alpha$ that decreases monotonically with $w$. In Table 1 we give estimates for the exponent $\alpha$ for several values of $w$. We have also checked for several values of $w$ that the scaling law

$$
G(x, L)=L^{1-\alpha} g(x, L)=G\left(\frac{x}{L}\right)
$$

holds.

From this investigation one can conclude that for values of $w>0.05$ and maybe smaller, the system stays scale invariant with a varying critical exponent. In the next sections we are going to show that this picture stays valid.

$\S$ For the appearance of operators with conformal spin in the presence of the quantum group symmetry mentioned in Appendix A, see Ref. [20]. 


\begin{tabular}{|c|c|c|c|c|c|c|c|c|}
\hline $\mathrm{w}$ & 1 & 0.85 & 0.75 & 0.4 & 0.25 & 0.1 & 0.05 & 0.025 \\
\hline$\alpha$ & 0.67 & 0.50 & 0.40 & 0.30 & 0.24 & 0.15 & 0.06 & 0.01 \\
\hline
\end{tabular}

Table 1. Estimates of the exponent $\alpha$ giving the increase of the number of clusters with the size of the system $L$.

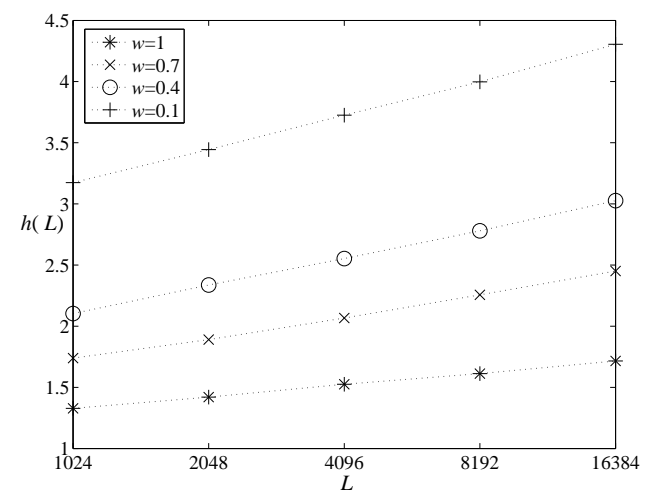

Figure 8. Average height $h(L)$ as a function of the lattice size $L$ for various values of $w \geq 0.1$.
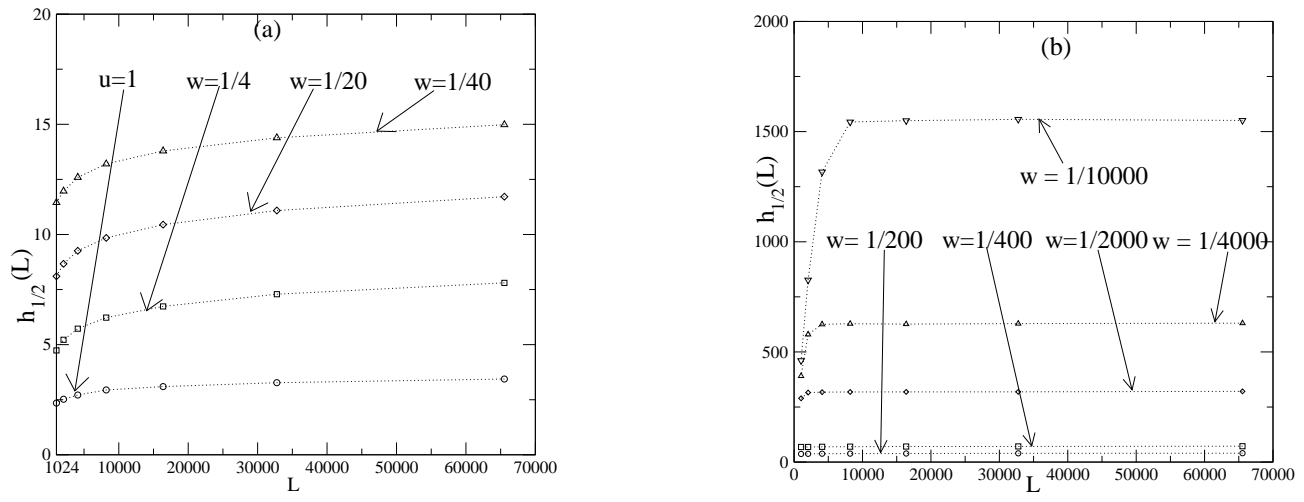

Figure 9. (a) Average height in the middle of the lattice $h_{\frac{1}{2}}(L)$ as a function of the lattice size $L$ for some values of $w$ in the range $1 \geq w \geq 0.025$. (b) Average height in the middle of the lattice $h_{\frac{1}{2}}(L)$ as a function of the lattice size $L$ for various small values of $w(w \leq 1 / 200)$.

Although it seems obvious that one should look at the $L$ dependence of the average value of the heights $h(L)$ or the average value of the height at the middle of the lattice $h_{\frac{1}{2}}(L)$, it turns out that these quantities are harder to study since they vary slowly with the system size. In Fig. 8 we show $h(L)$ for various values of $w \geq 0.1$. One sees a slow (logarithmic ?) increase when $L$ increases. A similar picture is obtained if one considers $h_{\frac{1}{2}}(L)$ (Fig. 9](a)).

A different picture emerges if one considers very small values of $w$ as shown in Fig. 9(b). One can observe two different phenomena. The values of $h_{\frac{1}{2}}(L)$ increase substantially as compared to the values observed at larger values of $w$, and more interestingly, it looks like they saturate for large values of $L$. Two scenarios are 


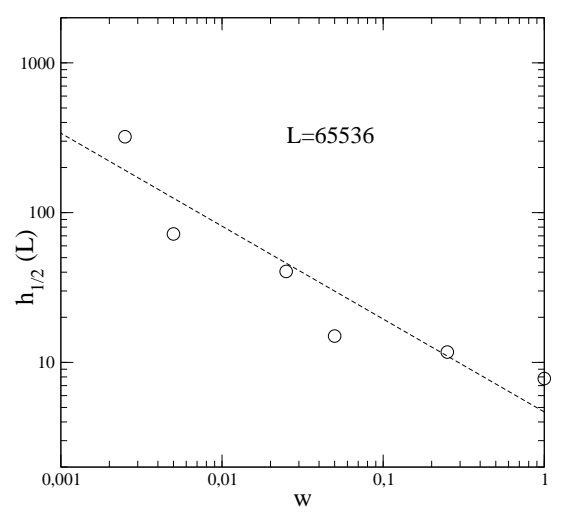

Figure 10. Average height in the middle of the lattice $h_{\frac{1}{2}}(L)$ as a function of $w$ for the lattice size $L=65536$. The dashed curve is given by 14.

compatible with our last observation. There is a change of the physics which occurs at very small values of $w$ illustrated by a change of the behavior of the $L$ dependence of $h_{\frac{1}{2}}(L)$. This is the first scenario. If this would be the case, we would have observed a crossing of the increasing functions $h_{\frac{1}{2}}(L)$ obtained for larger values of $w$ and the flat values of $h_{\frac{1}{2}}(L)$ observed for small values of $w$. This is not the case. What looks more plausible is the second scenario where for larger values of $w, h_{\frac{1}{2}}(L)$ also saturates but this is seen only at larger values of $L$ than those considered in our simulations.

According to the second scenario, for all values of $w<1$ (maybe including $w=1$ ) and very large values of $L, h_{\frac{1}{2}}(L)$ saturates. In order to check if this scenario makes sense, taking $L=65536$ we have done a fit to the curve which gives the maximum values of $h_{\frac{1}{2}}(L)$ (denoted by $h_{\max }$ ) for various values of $w$. One obtains:

$$
h_{\max }=\frac{4.67}{w^{0.62}} .
$$

As seen in Fig. 10 one obtains a fair fit to the data. Interestingly, if we use (14) for $w=1$ we obtain $h_{\max }=4.67$, a value compatible with the results shown in Fig. 9(a). Taking the results shown in Fig. 9(b) at face value, implies that the phase SOC (see Fig. 4) extends at least to the value $w=10^{-4}$. On the other hand for $w=0$ the single configuration is the full triangle, $h_{\max }$ has values of the size of the system and therefore the $L$-dependence of $h_{\max }$ shows no saturation. This suggests a nonanalytical behaviour of various quantities describing the system at $w=0$. In order to illustrate this point, in Fig. [11 we show typical configurations in the stationary state for a very small value of $w(w=0.00025)$ and different system sizes. One can see a clear change in the role of the boundaries if $L$ varies from 1024 to 32768 . For $L=1024$ one is close to the full triangle configuration (expected for $w=0$ ). However as one increases $L$ one gets a plateau (in which the height is almost constant and $L$ independent) and the boundaries play a less and less important role.

\section{Space-time phenomena}

In the last section, in which we have studied the properties of the stationary states, the data have confirmed the phase diagram shown in Fig. 4. In the present section 


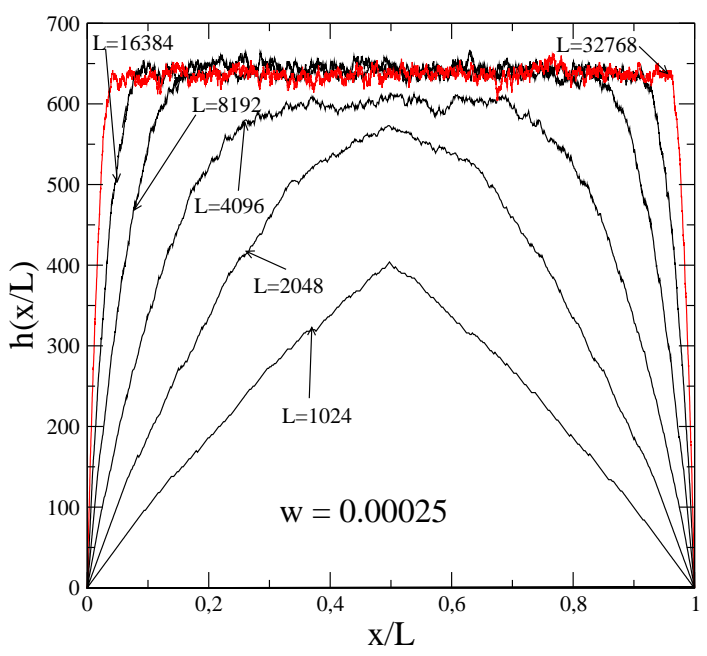

Figure 11. Typical configurations in the stationary state for $w=0.00025$ and different values of $L$.

we are presenting new facts which suggests that our assumption is correct. We are going to use the Family-Vicsek [21] scaling in order to check if the system is in a scale invariant phase and if the answer is positive, determine the dynamical critical exponent $z$. If we consider a time-dependent average quantity $a(t, L)$, being $a(L)$ the average in the stationary state, than for large values of $t$ and $L$ the function $A(t, L)$ scales as:

$$
A(t, L)=\frac{a(t, L)}{a(L)}-1 \sim A\left(\frac{t}{L^{z}}\right),
$$

where $z$ is the dynamical critical exponent. The scaling function depends on the initial conditions. In the data shown below, we have taken as initial condition the substrate $(h(2 i)=0, h(2 i+1)=1,(i=0, \ldots, n))$ with probability one. We first consider the quantity $K(t, L)$ which corresponds to the average number of clusters $(a(t, L)$ in (15) is $k(t, L)$ in this case).

In Fig. [12 (a) we show the function $K(t, L)$ for $u=0.4$ for different lattice sizes. We notice that for large lattice sizes, $K(t, L)$ is a function on $t$ only and can be obviously fitted with a sum of exponential functions as expected in a massive phase. Fig. 12(b) shows $K(t, L)$ for $u=1$ and as expected from conformal invariance, $z=1$. Conformal invariance gives also information on the function $K(t, L)$. One can use the knowledge of the finite-size scaling limit spectrum and obtain the functional dependence of $K(t / L)$. To illustrate how it works (see Fig. 12(b)), for large values of $\mathrm{t} / \mathrm{L}$ one can do a fit to the data, using the following ansatz:

$$
K(t / L)=C_{1} e^{-\frac{\lambda_{1} t}{L}}+C_{2} e^{-\frac{\lambda_{2} t}{L}},
$$

where the $\lambda_{i}$ are obtained from the spectrum of the Hamiltonian (A.7),

$$
E_{i}=\frac{\lambda_{i}}{L}, i=1,2 .
$$

The constants $C_{i}$ depend on the initial conditions. If one starts with the substrate $C_{1}=0.28$ and $C_{2}=0.54$. 

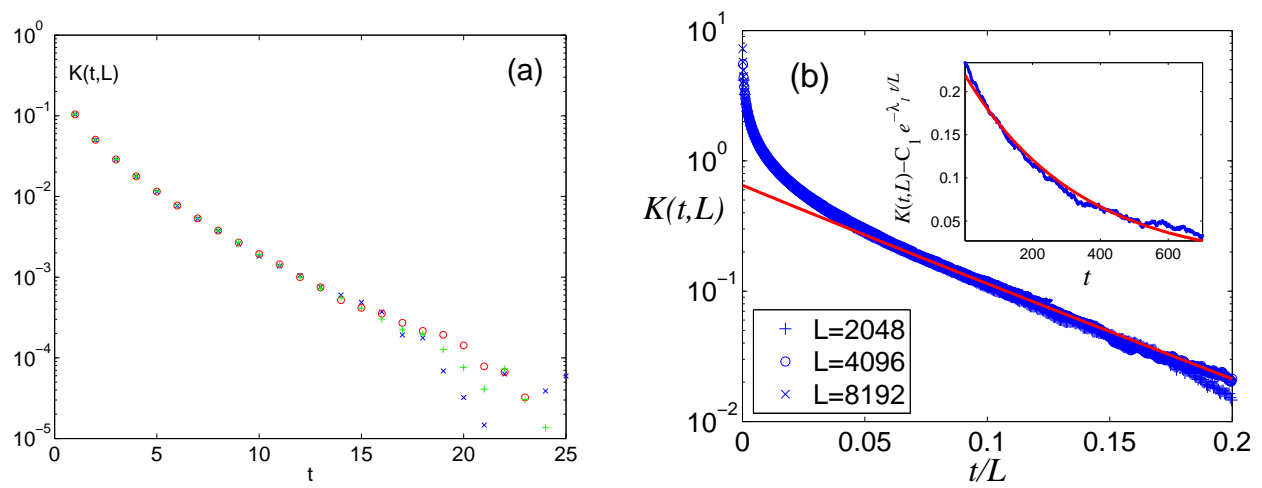

Figure 12. (a) Function $K(t, L)$, defined on [15), for $u=0.4$ as a function of time for the lattice sizes $L=2048,4096$ and 8192. (b) Function $K(t, L)$ for $u=1$ as a function of $t / L$ for various lattice sizes. Red curve is given by a fit to (16) considering only the first exponential with $\lambda_{1}=\pi \frac{9}{2} \sqrt{3}$ and $C_{1}=0.18$. In the inset we compare $K(t, l)-C_{1} e^{-\lambda_{1} t / L}$ for $L=8192$ with the short time exponential $C_{2} e^{-\lambda_{2} t / L}$ (given in red line) with $\lambda_{2}=\frac{3}{2} \lambda_{1}$ and $C_{2}=0.54$.

\begin{tabular}{|c|c|c|c|}
\hline$w$ & $K$ & $H$ & $H_{1 / 2}$ \\
\hline 1 & 1.03 & 0.95 & 0.97 \\
0.9 & 0.86 & 0.74 & 0.72 \\
0.7 & 0.55 & 0.52 & 0.50 \\
0.4 & 0.35 & 0.34 & 0.34 \\
0.25 & 0.31 & 0.30 & 0.30 \\
0.025 & $*$ & 0.07 & 0.07 \\
\hline
\end{tabular}

Table 2. Estimates of the dynamical critical exponent $z$ for various values of $w$. The estimates were obtained using 15 with $A$ replaced by $K, H$ and $H_{\frac{1}{2}}$. The number represented by $\left(^{*}\right)$ is not reliable since in that region the number of clusters is very small.

We now consider smaller values of $w$ (larger values of $u$ ). We have done an extensive study of various functions $A(t, L)$ taking $k(t, L), h(t, L)$ and $h_{\frac{1}{2}}(t, L)$ in (15). The estimated values for the critical exponent $z$ are shown in Table 2. For very small values of $w$, the estimates coming from $K(t, L)$ are poor because the number of clusters is small and hence large errors.

Inspection of Table 2 shows a smooth drop of the values of $z$ from $z=1$ for $w=1$ to $z=0$ for $w=0$. The latter values is in agreement with a direct calculation of the mass gap at $w=0[1]$ which gives a finite gap in the thermodynamic limit. To show how the estimates given in Table 2 work, we demonstrate in Figs. 133 (a) and 13 (b) the scaling functions $K(t, L)$ for $w=0.4$ and $H_{\frac{1}{2}}(t, L)$ for $w=0.0025$, respectively.

We have not studied extensively the two-contact points space-time correlation functions except for the special case $u=1$. This case is interesting since from conformal invariance the two-contact points space-time correlation function exhibits, far from the boundaries, the following scaling form in the bulk (see (91)):

$$
C(R, t, L)=L^{-\frac{2}{3}} G(\mu),
$$



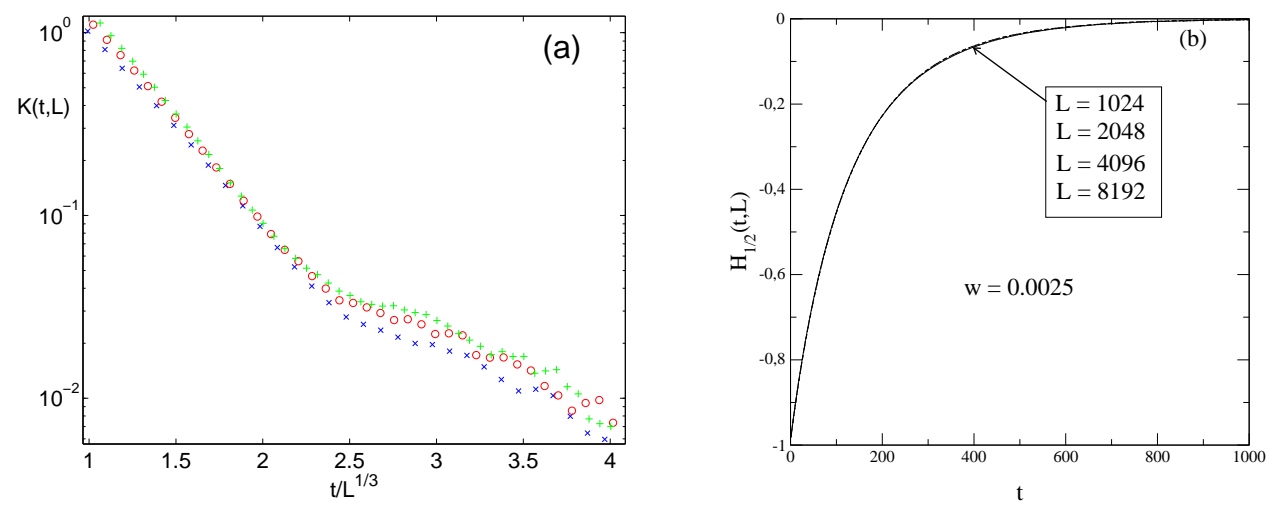

Figure 13. (a) $K(t, L)$ for $w=0.4$ as a function of $t / L^{\frac{1}{3}}$ for the lattice sizes $L=1024,2048,4096$ and 8196. (b) $H_{\frac{1}{2}}(t, L)=h_{\frac{1}{2}}(t, L) / h_{\frac{1}{2}}(L)-1$ for $w=0.0025$ as a function of $t$ for various lattice sizes.

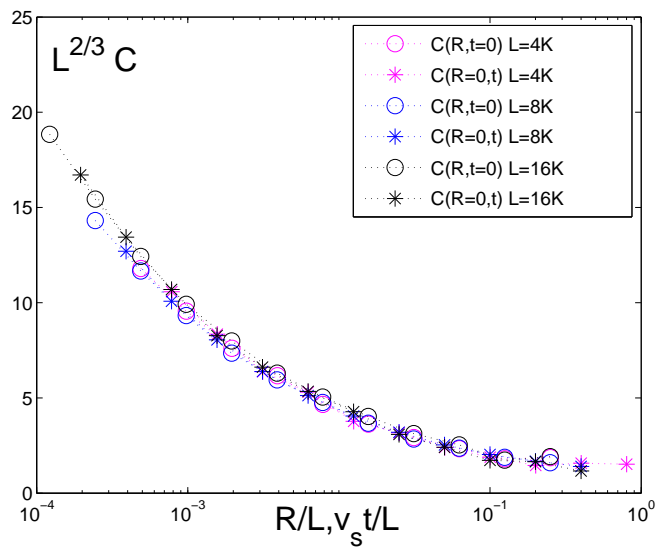

Figure 14. The 2-contact point correlation function $C(R, t, L)$ times $L^{\frac{2}{3}}$ as a function of the scaled distance $R / L$ and the scaled time $\frac{v_{s}}{L} t\left(v_{s}=\frac{3}{2} \sqrt{3}\right.$ is the sound velocity) for various lattice sizes.

where

$$
\mu=\frac{\sqrt{R^{2}+v_{s}^{2} t^{2}}}{L} .
$$

In (18) and (19) $R$ is the distance between the contact points, $t$ is the time difference and $v_{s}=\frac{3}{2} \sqrt{3}$ is the sound velocity (see Appendix A). The factor $L^{-\frac{2}{3}}$ in (18) is obtained from (9). In Fig. 14] we show the scaling function $G(\mu) \|$. To avoid boundary effects the data was taken only in the center segment of size $L / 2$.

The functional dependence (18) which is special for the conformal invariant case is nicely seen in Fig. 14. Our data are not good enough in order to extract from the small $\mu$ behavior of $G(\mu)$, the two-contact point correlation function in the thermodynamic limit.

\| We have not used the connected correlation function in (18). 


\section{Avalanches in the RPM}

An interesting property of the RPM is the occurrence of avalanches in the desorption processes. For the case $u=1$, based on exact results on small lattices, the existence of avalanches was already suggested in [1]. In this section, using Monte Carlo simulations on large lattices, we present the properties of avalanches in the whole domain $0<w \leq 1$.

In the stationary state, once a tile from the rarefied gas hits the interface, it can be reflected, adsorbed or can trigger a nonlocal desorption process in which many tiles may leave the interface, this defines an avalanche. In the latter case the size of the avalanche is given by the number of tiles $T$ that are released in the process. This number is always odd, therefore it is convenient to write: $T=2 v-1(v=1,2, \ldots)$. For $u<1$ only a finite number of tiles are removed, since the density of clusters is finite. For $w \leq 1$, the cluster density vanishes, and therefore macroscopic number of tiles may be desorbed, hence macroscopic avalanches.

We denote by $S(v, L)$ the PDF which gives the probability for an avalanche of size $v$ for a system of size $L$. In models of self-organized criticality (SOC) $[5,11,22-24]$, for large values of $v$ and $L$, one may expect the PDF to exhibit a simple scaling law,

$$
S(v, L)=v^{-\tau} F\left(\frac{v}{L^{D}}\right),
$$

characterized by two exponents $\tau$ and $D$.

It is convenient to consider moments of the PDF and their scaling properties:

$$
<v^{m}>_{L}=\sum_{v=1} v^{m} S(v, L)=\Gamma_{m} L^{\sigma(m)}
$$

where

$$
\sigma(m)=\left\{\begin{array}{cc}
0, & m<\tau-1 \\
D(m+1-\tau), & m>\tau-1
\end{array} .\right.
$$

In order to get estimates for $D$ and $\tau$ from the Monte Carlo simulations, it is convenient to take couples of system sizes $L$ and $L^{\prime}$ and consider the quantities:

$$
\sigma_{L, L^{\prime}}(m)=\ln \left(\frac{\left\langle v^{m}\right\rangle_{L}}{\left\langle v^{m}\right\rangle_{L^{\prime}}}\right) / \ln \left(\frac{L}{L^{\prime}}\right)=m D_{L, L^{\prime}}+B_{L, L^{\prime}}
$$

and

$$
\sigma_{L, L^{\prime}}\left(m_{L, L^{\prime}}\right)=0, \quad \tau_{L, L^{\prime}}=m_{L, L^{\prime}}+1
$$

Using the results of Monte Carlo simulations for couple of sizes (in practice we have chosen $L^{\prime}=2 L$ ) one can obtain estimates for the two critical exponents $D$ and $\tau$ from $\sigma_{L, 2 L}$ and $\tau_{L, 2 L}$. In Fig. 15 we illustrate the $m$ dependence of $\sigma_{L, 2 L}$ obtained from Monte Carlo simulations, for several values of $L$ in the $u=1$ case.

From a cursory look at this figure one can see that for $u=1, \tau \approx 3$ and $D \approx 1$ confirming the results obtained in [1] using much smaller lattices (for a more precise estimate, see Table 3). The result $D=1$ is to be expected since in a conformal invariant theory (this is the case if $u=1$ ) one has no other length than the size of the system $L$. We found no explanation for the value three of $\tau$ although this number shows up in many aspects of the model (see for example (9) and the Appendix). In Fig. 16] we show the scaling function $F(v / L)$ defined in (20). One observe a nice data collapse.

In Table 3 we give the estimates for the exponent $\tau$ and $D$ for various values of $w$. The estimates presented in this Table are obtained using only one pair of sizes (4096 


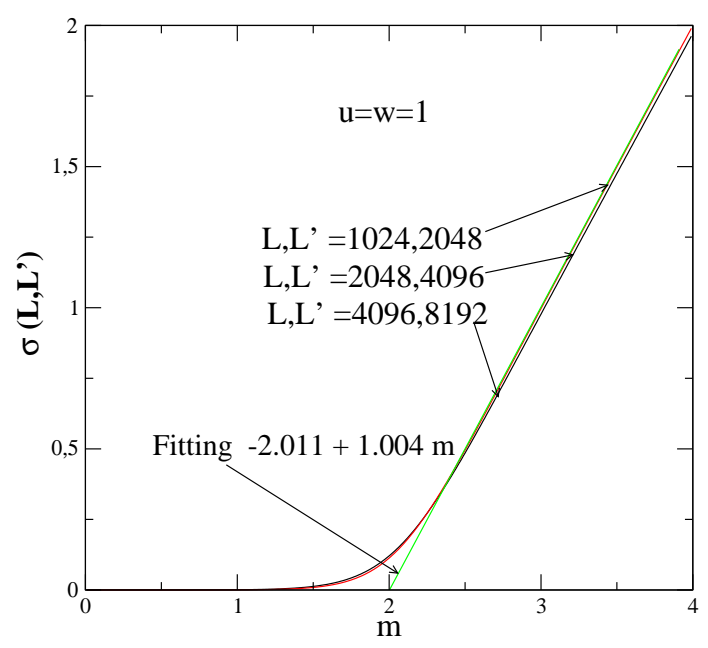

Figure 15. The $m$ dependence of $\sigma_{L, 2 L}$ for $w=1$. The fitted straight line is also shown.

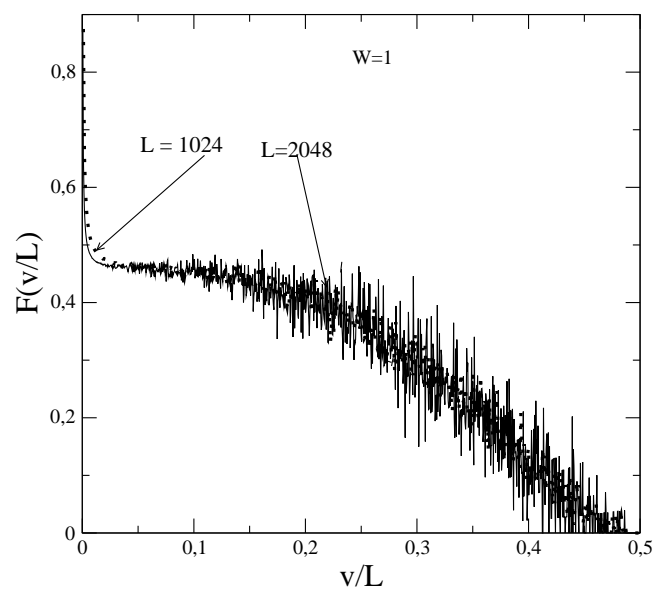

Figure 16. The scaling function $F(v / L)$ for $w=1$. The data are for lattice sizes $L=1024$ and $L=2048$.

and 8192) but we made sure that the results are reliable by studying the changes of the values of the estimates using smaller lattices.

For small values of $w$, the determination of $\tau$ has to be done with care. This is due to surface effects (there are avalanches occurring at both ends of the system which have a different behaviour than avalanches occurring in the bulk). The phenomenon is illustrated in Fig. 17(a) where the PDF $S(v, L)$ is shown for $w=0.00025$ and $L=32768$. One notices that $S(v / L)$ has two peaks: one for $v=1$ and another one for very large values of $v$. Large avalanches occur when one tile hits the boundary of the interface. On the other hand, one expects that for large values of $L$, the probability for a tile to hit a boundary get smaller and smaller as the system gets larger (see Fig. 11), therefore the probability to have large avalanches should decrease with the size of the system. This phenomenon is nicely illustrated in Fig. 17(b), where one can 
Conformal invariance and its breaking
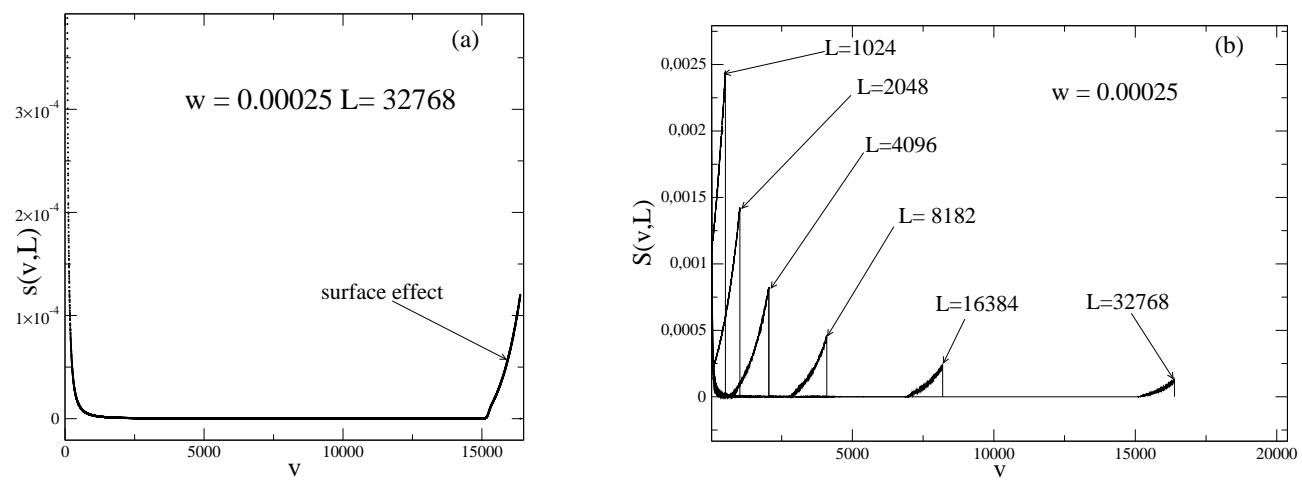

Figure 17. (a) The PDF for avalanches for $w=0.00025$ and $L=32768$. (b) The PDF of avalanches for $w=0.00025$ and different sizes.
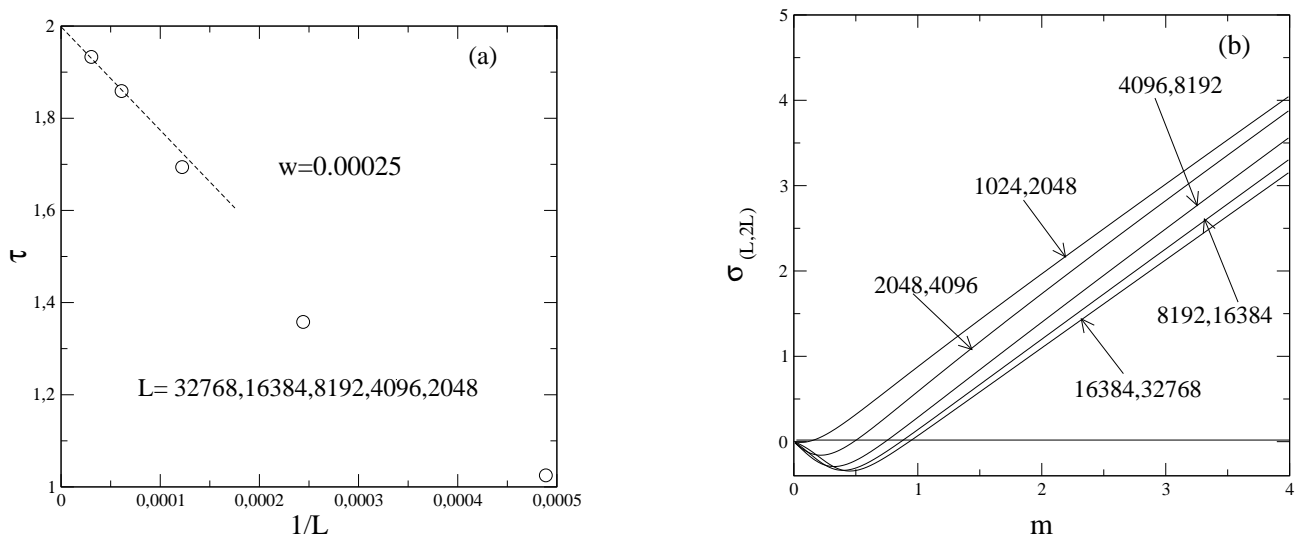

Figure 18. (a) Estimates $\tau_{L, 2 L}$ for $w=0.00025$ using different values of $L$ from $L=2048$ to $L=16384$ (b) The $m$ dependence of $\sigma_{L, 2 L}$ for $w=0.00025$.

see that the probability to have an avalanche at the boundary decreases with the size of the system. One expects that the determination of $\tau$ using (23) and (24) will give a smaller value for $\tau$ for small lattices (large avalanches at the boundaries) and a larger value when compared with the value obtained by using large lattices (the importance of the boundary effects decreases). That is exactly what happens as can be seen in Fig. 18(a). One sees in this figure that the estimates for $\tau$ converge to a value close to 2 .

In order to illustrate the convergence of the estimates in Fig. 18(b) we present for a very small value of $w$ the functions $\sigma_{L, 2 L}$ for several values of $L$. Although the convergence is poorer when compared with these quantities obtained for higher values of $w$ (see Fig. 15), one can still get reliable results.

The value of the exponent $D$ stays unchanged and equals to one in the whole range $w<1$. This can be understood in the following way [25] the tiles which are desorbed and create the avalanche belong always to a one-dimensional layer.

We turn now to the exponent $\tau$. As can be seen in Table 3 this exponent varies from the value $3(u=1)$ to a value close to 2 for $w$ close to zero. In the case $u=1$, the average size of the avalanches stays finite when $L$ gets large but the dispersion 


\begin{tabular}{|c|c|c|c|c|c|c|c|c|c|c|}
\hline$w$ & 1 & 0.95 & 0.90 & 0.80 & 0.60 & 0.45 & 0.30 & 0.05 & 0.02 & 0.005 \\
$D_{L, L^{\prime}}$ & 1.004 & 1.089 & 1.087 & 1.066 & 1.040 & 1.026 & 1.017 & 1.002 & 1.002 & 1.006 \\
$\tau_{L, L^{\prime}}$ & 3.00 & 2.77 & 2.63 & 2.47 & 2.32 & 2.25 & 2.18 & 2.07 & 2.046 & 2.00 \\
\hline
\end{tabular}

Table 3. Estimates of the critical exponents $D$ and $\tau$ obtained by using lattices sizes $L=4096$ and $L^{\prime}=8196$.

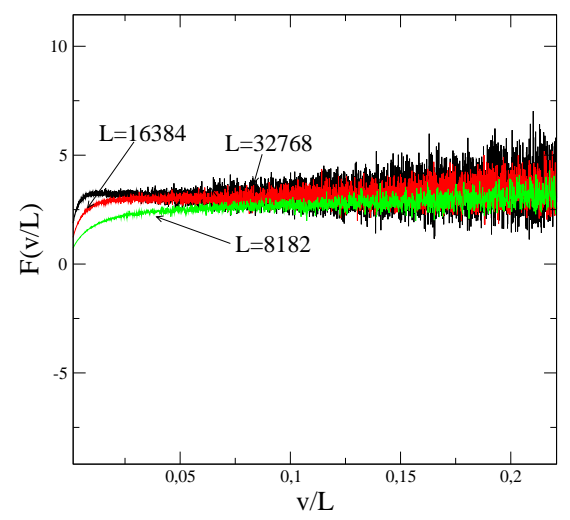

Figure 19. The functions $F(v / L)$, defined on 20, at $w=0.00025$ for some values of $L$.

diverges (the function $F(v / L)$ shown in Fig. [16] does not vanish at the origin). Does an exponent $\tau=2$ mean that the average size of the avalanches diverges logarithmically with $L$ ? Not necessarily, it depends if the scaling function $F(v / L)$ vanishes or not at the origin. In Fig. 19] we show for $w=0.00025$, that $F(v / L)$ does not vanish at the origin. Since later in this section we are going to show that $\langle v\rangle$ stays finite for any $w>0$, we conclude that $\tau$ gets very close to the value 2 but never reaches it.

We would like to comment now on the properties of the avalanches seen at the boundaries and illustrated in Fig. 17. For large values of $v$ where the boundary effects occur, the PDF $S(v, L)$ presents two striking features: the probability to have an large avalanche decreases with $L$ and $S(v, L)$ increases with $v$. A close inspection of the local heights distribution for different values of $L$ seen in Fig. 11] allows us to investigate both features. Firstly, one sees that for large values of $L$ the boundary becomes less and less important and therefore the probability for a tile to hit the boundary and produce a large avalanche decreases. On the other hand, at the boundary, the probability for a hit by a tile is proportional to $|d h(x, L) / d x|$ (keep in mind that a desorption process takes place when the local slope $s(i)$ takes the values \pm 1 ). From the data shown in Fig. 11] one can see that for $x$ close to the boundaries, $d^{2} h(x, L) / d x^{2}$ is negative. This implies that the probability to hit the interface with a tile at the two ends of the system is larger than further into the system.

An important consequence of this study is that avalanches exist at least in the interval $1 \geq w \geq 0.00025$ and therefore the system is scale invariant in this interval.(The data shown in Tables 1 1 and 2 are limited to the interval $1 \geq w \geq 0.025$ ). It is probably safe to assume that the SOC phase covers the domain $1 \geq w \geq 0$. (We have obviously checked that for $u<1$, where the system is massive, for large values of $v$ the PDF has an exponential decrease and not an algebraic one).

We turn now our attention to the average size of the avalanches. A simple mean- 
field argument allows to compute it using informations about the stationary state only.

We first consider, in the stationary state, the average fraction of interface where desorption does not take place $n(L)$ (FND) defined in (6). This quantity varies from the value 1 for the substrate (the single configuration for $u=0$ ) to the value $1 /(L-1)$ for the full triangle (the single configuration for $w=0$ ).

For $u=1$ there is a conjecture [26] for the values of $n(L)$

$$
n(L)=\frac{3 L^{2}-2 L+2}{(L-1)(4 L+2)}=\frac{3}{4}\left(1-\frac{1}{6 L}+\cdots\right) \text {. }
$$

This conjecture was checked using our Monte Carlo simulations for various lattice sizes.

If one knows $n(L)$, the average probability $P_{a}(L)$ to have an adsorption process, the average probability $P_{r}(L)$ to have a reflection process and the average probability $P_{d}(L)$ to have a desorption process can be easily obtained:

$$
\begin{aligned}
& P_{a}(L)=\frac{n(L)}{2}-\frac{1}{2(L-1)}, \\
& P_{r}(L)=\frac{n(L)}{2}(2 w-1)+1-w+\frac{1}{2(L-1)}, \\
& P_{a}(L)+P_{r}(L)+P_{d}(L)=1,
\end{aligned}
$$

where, like in our Monte Carlo simulations, we choose $u_{a}=1$ and $u_{d}=w=1 / u$.

Taking into account that in the stationary state the average number of adsorbed tiles is equal to the average number of desorbed tiles, one obtains:

$$
\langle T\rangle_{L}=2\langle v\rangle_{L}-1=\frac{P_{a}(L)}{P_{d}(L)} .
$$

Notice that this is a mean-field calculation since we have first computed the average probability to have a desorption process and multiplied it with the average number of tiles which are desorbed. In the large $L$ limit (26)-(29) gives:

$$
\langle T\rangle_{\infty} \approx \frac{n_{\infty}}{2\left(1-n_{\infty}\right) w}
$$

In Fig. 20] one shows the $w$ dependence of $n_{\infty}$ as obtained from the extrapolated results of our Monte Carlo simulations. One notices a discontinuous behavior of $n_{\infty}$ around $w=0$. At $w=0$ one has $n_{\infty}=0$, while $\lim _{w \rightarrow 0_{+}} n_{\infty}=0.5$. Using (30) one concludes, in agreement with previous observations that for any finite value $w$, the average size of the avalanches is finite.

In Table 4 we compare in two cases the mean-field predictions for $\langle T\rangle_{L}$ given in (30) with the values measured in Monte-Carlo simulations. The agreement is excellent. In the same Table the values for $n(L)$ for several values of $L$ are also given. One notices that for $u=1$ and $L=1024$ one already gets within five digits the asymptotic value $3 / 4$. This is not the case for $w=0.00025$, where the finite-size effects are larger. Our numerical analysis indicate that the asymptotic behavior of $n(L)$ can be described by

$$
n(L)=n_{\infty}(1-A(w) / L+\cdots),
$$

where $A(1)=1 / 6$ and $A(w)$ diverges if $w \rightarrow 0$ (data not shown).

We conclude this section with the following observation, which is necessary for the consistency of the present model for avalanches. If one looks at the expression 


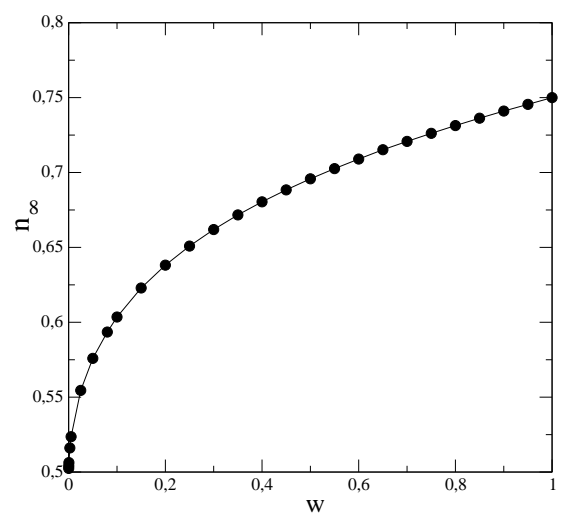

Figure 20. The $w$ dependence of the fraction of the interface where adsorption does not take place $n_{\infty}$ obtained from the extrapolated results of Monte Carlo simulations.

\begin{tabular}{|c|c|c|c|c|c|}
\hline$w$ & $L$ & $n_{L}$ & $\langle T\rangle_{L}$ & $\mathrm{MF}$ & $\langle T\rangle_{L} / \mathrm{MF}$ \\
\hline 1 & 1024 & 0.749 & 1.497 & 1.497 & 0.999 \\
1 & 2048 & 0.749 & 1.498 & 1.501 & 0.998 \\
1 & 4096 & 0.749 & 1.499 & 1.500 & 0.998 \\
1 & 8192 & 0.749 & 1.499 & 1.499 & 0.999 \\
\hline 0.00025 & 1024 & 0.199 & 496.999 & 496.119 & 1.001 \\
0.00025 & 2048 & 0.313 & 914.346 & 913.820 & 1.001 \\
0.00025 & 4096 & 0.407 & 1374.713 & 1373.856 & 1.001 \\
0.00025 & 8192 & 0.455 & 1674.877 & 1674.550 & 1.000 \\
\hline
\end{tabular}

Table 4. Values of $n_{\infty}$ and the average number of tiles obtained from Monte Carlo simulations $\langle T\rangle_{L}$ as compared with the compared with the mean-field results (MF). The values are given for several lattice sizes $L$ and two values of $w, w=1$ and $w=0.00025$.

(27) of the average (over configurations) probability that a tile hitting the interface is reflected, one sees that for small values of $w$, the probability approaches the value one. This implies that to obtain large avalanches ( $\operatorname{small} \tau$ ) one has to wait a long time. This is to be expected in models of SOC.

\section{Discussions}

The raise and peel model describes a stochastic process of a one-dimensional interface. RSOS paths give the configuration space. It is well known that if a stochastic model has as stationary state in which the RSOS paths have the same probability, the average height $\langle h(L)\rangle$ increases with the system size $L$ like $\sqrt{L}$ (Brownian motion). In the one-parameter ( $u$ or $w=1 / u$ ) RPM, in the stationary states, the various RSOS paths get weights and henceforth the physics is different. It turns out that for all finite values of $u(w \neq 0)$ in the large $L$ limit, $\langle h(L)\rangle$ stays finite. The convergence to the large $L$ limit of $\langle h(L)\rangle$ is very slow. The $w=0$ value is singular: in the stationary state one has, with probability one the full triangle and therefore $h=L / 2$. The phase diagram is shown in Fig. [4 For $u<1$, one is in the massive phase (finite correlation lengths and non-vanishing densities of clusters). For $1 \geq w>0$ the density of clusters 
vanishes algebraically with an exponent which depends on $w$ (Table 1). The dynamical exponent $z$ also depends on $u$. It varies between one and zero (see Table2). We called this phase the SOC phase since in the desorption process one has avalanches. The $\mathrm{PDF}$ of the number of tiles desorbed has long tails. Although the average number of tiles involved in the avalanches stays finite for large $L$, higher moments diverge. The $\tau$ exponent changes from the value $3(w=1)$ to the value 2 for $w$ close to zero (Table 3). The exponent $D$ is robust and keeps the value $D=1$. A fascinating aspect of the model is that, contrary to our intuition, for any small value $w>0$, if $L$ is large enough, the system separates into a bulk part and a "surface" part related to the condition that at the two boundaries the heights have to take the value zero (see Fig. [11).

The value $u=1$ is special. In the continuum time limit the Hamiltonian which gives the Markovian time evolution of the stochastic process, has the same spectrum as the XXZ quantum spin $1 / 2$ chain Hamiltonian with a given $Z$-anisotropy and fixed boundary terms (see Appendix A). This Hamiltonian is integrable and the finite-size scaling spectrum is known. The main observation is that for $u=1$ one has a spacetime symmetry (conformal invariance). There are several important consequences concerning the properties of the system. The one-point function which gives the local density of contact points has the scaling form given by (12). The two-contact points space-time correlation function has a scaling form given by (18). The dynamical critical exponent $z$ can be computed analytically and has the value 1 . There are other consequences of conformal invariance. The Family-Viczek scaling function which gives the time and size dependence of average quantities can also be obtained (see Fig. 12 $(\mathrm{b}))$. The value $D=1$ is a consequence of the fact that in a conformal theory, in the large $L$ limit the single scale is $L$ itself. The value $\tau=3$ remains a puzzle although the number 3 appears all over for $u=1$ (see Appendix A).

The reader not familiar with conformal invariance might skip the end of this section. We have shown that the density of contact points gives a local operator of a $c=0$ ( $c$ is the central charge of the Virasoro algebra) conformal field theory. As discussed in Section 3 the value of the scaling dimension of this operator has not a simple explanation. Other local operators can be defined in a similar way. For example one can look at the local density of points where one has a deep (slope zero) at the height 1 (or any fixed height).

One interesting aspect of the RPM is that one has a simple example of an unusual kind of conformal symmetry breaking. For $u<1$ the system becomes massive. This is not a surprise. One can understand why this happens in the following way: one can take the desorption rate $u_{d}=1$ (see Section 2) and $u_{a}=u$. This implies that the perturbation is local (the adsorption is local). If $w<1$ the situation is quite different

and here comes the surprise. One has scale invariance without having conformal invariance. The appearance of this novel phenomenon has the following explanation. For $w<1$ one takes $u_{a}=1$ and $u_{d}=w$. Desorption is a nonlocal process and therefore the conformal theory is perturbed nonlocally and there is no a priori reason why conformal invariance cannot be broken while scale invariance is not.

\section{Acknowledgements}

We would like to thank the referee for pointing out an embarrassing mistake in Section 3. It is our pleasure to acknowledge J. L. Cardy, B. Schittmann and D. Dhar for reading this manuscript and useful discussions. We also thank M. Bauer, J. 
de Gier and M. Flohr for related discussions. FCA research was supported in part by FAPESP and CNPq (Brazilian Agencies). Research of EL was supported by the NSF PFC-sponsored Center for Theoretical Biological Physics (Grants No. PHY0216576 and PHY-0225630). VR gratefully acknowledges support from the Deutsche Forshungsgemeinschaft, The Australian Research Council, the EU network HPRNCT-2202-00325 and FAPESP (Brazil).

\section{Appendix A. The finite-size scaling limit of the spectrum of the Hamiltonian for $u=1$. Results from conformal field theory.}

As shown in Ref. [1], in continuum time, the time evolution of the RPM for $u=1$, is given by the Hamiltonian:

$$
H=\sum_{i=1}^{L-1}\left(1-e_{i}\right),
$$

where $e_{i}$ acts on the configurations (RSOS paths) described in Section 1, taking one path into another or leaving it unchanged. Using a similarity transformation from the configuration basis to a spin basis, the $e_{i}$ 's have a representation in terms of Pauli matrices:

$$
e_{i}=\frac{1}{2}\left[\sigma_{i}^{x} \sigma_{i+1}^{x}+\sigma_{i}^{y} \sigma_{i+1}^{y}-\frac{1}{2} \sigma_{i}^{z} \sigma_{i+1}^{z}+\frac{1}{2}+i \frac{\sqrt{3}}{2}\left(\sigma_{i}^{z}-\sigma_{i+1}^{z}\right)\right] .
$$

If we introduce the expressions A.2 in A.1 one obtains a well known Hamiltonian of an integrable quantum chain acting into a $2^{L}$ dimensional vector space. This chain has $U_{q}(s l(2))$ symmetry with $q=\exp (i \pi / 3)$. To obtain the action of $H$ in the vector space of the RSOS paths one has to consider only the subspace of $U_{q}(\operatorname{sl}(2))$ scalars ( $\operatorname{spin} s=0$ ) which has the dimension $C_{n}=(2 n) ! /(n+1)(n !)^{2}$ with $L=2 n$. The spectrum of the Hamiltonian (A.1) is non-negative. Let us denote by $E_{r}(r=0,1, \ldots)$ the energy levels in increasing order:

$$
E_{0}=0<E_{1}<E_{2}<\cdots .
$$

The partition function giving the finite-size limit of the spectrum of the Hamiltonian of the quantum chain, is defined as follows:

$$
Z(q)=\lim _{L \rightarrow \infty} Z_{L}(q)=\lim _{L \rightarrow \infty} \sum_{n=0} q^{\frac{L E_{n}}{\pi v_{s}}}
$$

where $v_{s}=3 \sqrt{3} / 2$. One can show [13] that $Z(q)$ has the expression

$$
Z(q)=\sum_{s=0}^{\infty}(2 s+1) \xi_{s}(q)
$$

where

$$
\xi_{s}(q)=q^{\frac{s(2 s-1)}{3}}\left(1-q^{2 s+1}\right) \prod_{n=1}^{\infty}\left(1-q^{n}\right)^{-1}
$$

is the partition function in the spin $s$ sector.

In Section 4 we look at the time evolution of the stochastic model. The finite size-scaling limit is given by a.6 with $s=0$. This implies that in the large $L$ limit we have

$$
L E_{0}=0, \quad L E_{1}=\pi 3 \sqrt{3}, \quad L E_{2}=\pi \frac{9}{2} \sqrt{3}, \quad \ldots .
$$


Up to now we have described the time evolution of the system. If one considers space-time correlation functions, the various critical exponents are expected to be obtained $[27,28]$ from the scaling dimensions of the primary fields of minimal models [29] with a central charge $\mathrm{c}=0[1]$ :

$$
\Delta=\frac{(3 p-2 q)^{2}-1}{24}
$$

where $p$ and $q$ are positive integers. Notice that the value $\Delta=1 / 3$ appears in both the finite-size spectrum of $H$ (see (A.6) with $s=1$ ) and in (A.8).

\section{References}

[1] J. de Gier, B. Nienhuis, P. Pearce and V. Rittenberg, J.Stat.Phys. 114 (2004) 1.

[2] J. de Gier, B. Nienhuis, P. A. Pearce and V. Rittenberg, Phys.Rev. E 67, 016101 (2002).

[3] J. de Gier, B. Nienhuis, P. A. Pearce and V. Rittenberg, J.Phys. A 34, L265 (2001).

[4] D. M. Bressoud 1999 Proofs and Confirmations: The story of the Alternating Sign Matrix Conjecture (Cambridge: Cambridge Press).

[5] D. Dhar, Physica A 263 (1999) 4.

[6] J. Polchinski, Nucl.Phys. B 303 (1988) 226.

[7] V. Riva and J. L. Cardy, hep-th/0504197

[8] P. C. Hohenberg and B. I. Halperin, Rev. Mod. Phys. 49 (1977) 435.

[9] J. L. Cardy, cond-mat/9312032

[10] P. F. Arndt and T. Heinzel, J.Phys. A 28 (1995) 3567.

[11] C. Tebladi, M. De Menech and A. L. Stella, Phys.Rev.Lett. 83 (1999) 3952.

[12] V. Pasquier and H. Saleur, Nucl.Phys.B 330 (1990) 523.

[13] M. Bauer and H. Saleur, Nucl.Phys. B 320 (1989) 591.

[14] J. de Gier, arXiv:math. CO/0211285 (2002).

[15] T. W. Burkhardt and T. Xue, Phys.Rev.Letters 66 (1991) 895.

[16] M. Flohr, Private Communication.

[17] P. Bouwknegt and K. Schoutens, Phys. Rep. 223 (1993) 183.

[18] M. Bauer, Private Communication.

[19] M. Bauer and D. Bernard, Commun. Math. Phys. 239 (2003) 493.

[20] P. F. Arndt, T. Heinzel, J.Phys. A 28 (1995) 3567.

[21] F. Family and E. Vicsek, J.Phys.A 18 (1985) L75.

[22] P. Bak, C. Tang and K. Wiesenfeld, Phys.Rev. A 38 (1088) 364.

[23] D. Dhar and R. Ramaswamy,Phys.Rev.Lett. 63 (1989) 1659.

[24] V. B. Priezzhev, E. V. Ivashkevich, A. M. Povolotsky and Chin-Kun Hu, Phys.Rev.Lett. 87 (2001) 084301.

[25] D. Dhar, Private Communication.

[26] Yu. G. Stroganov, Private Communication.

[27] F. C. Alcaraz, M. N. Barber, M. T. Batchelor, Ann. Phys. (USA) 182 (1988) 280.

[28] F. C. Alcaraz, U. Grimm and V. Rittenberg, Nucl. Phys. B316 (1989) 735.

[29] C. Itzykson, H. Saleur and J. -B. Zuber (1988) Conformal invariance and applications to statistical mechanics (Singapore:World Scientific). 